\title{
Patient Safety in Ambulatory Pediatrics
}

\author{
Kathleen Huth, MD, MMSC \\ Arda Hotz, MD, MPH \\ Amy J. Starmer, $M D, M P H^{*}$
}

\author{
Address \\ *Department of Pediatrics, Boston Children's Hospital, Boston, MA, USA \\ Email: amy.starmer@childrens.harvard.edu
}

Published online: 14 0ctober 2020

(C) Springer Nature Switzerland AG 2020

This article is part of the Topical Collection on Patient Safety

Keywords Patient safety $\cdot$ Medical errors $\cdot$ Ambulatory care

\begin{abstract}
Purpose of Review The majority of patient care occurs in the ambulatory setting, and pediatric patients are at high risk of medical error and harm. Prior studies have described various safety threats in ambulatory pediatrics, and little is known about effective strategies to minimize error. The purpose of this review is to identify best practices for optimizing safety in ambulatory pediatrics.

Recent Findings The majority of the patient safety literature in ambulatory pediatrics describes frequencies and types of medical errors. Study of effective interventions to reduce error, and particularly to reduce harm, have been limited. There is evidence that medical complexity and social context are important modifiers of risk. Telemedicine has emerged as a care delivery model with potential to ameliorate and exacerbate safety threats. Though there is variation across studies, developing a safety culture, partnerships with patients and families, and use of structured communication are strategies that support patient safety.

Summary There is no standardized taxonomy for errors in ambulatory pediatrics, but errors related to medications, vaccines, diagnosis, and care coordination and care transitions are commonly described. Evidence-based approaches to optimize safety include standardized prescribing and medication reconciliation practices, appropriate use of decision support tools in the electronic health record, and communication strategies like teach-back. Further high-quality intervention studies in pediatric ambulatory care that assess impact on patient harm and clinical outcomes should be prioritized.
\end{abstract}




\section{Introduction}

The majority of pediatric care occurs outside of the hospital, yet there is a relative lack of data on medical error or patient harm in the ambulatory care setting. Preventing harm caused by clinical care is the central tenet of patient safety, a key healthcare quality domain identified by the Institute of Medicine [1]. Prior reviews have summarized patient safety concerns in ambulatory care [2], and more recently, there has been a call to prioritize ambulatory patient safety as a focus for further research [3]. With the growing complexity of pediatric care as well as evolution in how ambulatory care is delivered, pediatricians face new challenges as well as new opportunities for ensuring safe care.

This article reviews the epidemiology of key categories of safety threats in ambulatory pediatrics. We then describe best practices to minimize each category of errors, in order to maximize patient safety within this context. We also review key contextual factors that have potential to either ameliorate or exacerbate these threats to safety, and offer a conceptual framework summarizing key themes and considerations related to pediatric patient safety in ambulatory care.

\section{Ambulatory pediatric errors and interventions to prevent error}

The literature on error type epidemiology in ambulatory pediatrics is limited, and complicated by a lack of consistency with which errors are categorized. Major error types relevant to ambulatory pediatrics include medication errors, vaccine errors, diagnostic errors, and errors related to miscommunication during care coordination and transitions of care. A summary of error types and subtypes relevant to ambulatory pediatrics is provided in Table 1, with a description of interventions that have been proposed and studied in an effort to prevent errors and reduce harm (Table 2).

Medication errors are the most common type of errors experienced by pediatric patients $[4,5]$. Children are at higher risk of medication errors than adults due to challenges with weight-based dosing, frequent use of liquid formulations with variability in compounding practices, and lack of standards for medication preparation and dosing [6-8]. There is a risk of confusion between look-alike and sound-alike medications, complicated by lack of standardized medication packaging and labelling [9]. Polypharmacy is a major risk factor for adverse drug events in children [10] and is relatively common: based on a 2009 survey, more than half of children in the USA used one or more medications in the prior week [11]. Multiple caregivers may be involved in medication administration in the home and at school which could increase opportunity for error. Specific characterization of pediatric medication errors in the ambulatory setting is underrepresented in the literature, suggesting an opportunity for further research [12•].

Errors may occur when prescribing, dispensing, administering, or monitoring effects of medications [1]. In the prescribing phase, inappropriate abbreviations have been reported as a major source of error in the ambulatory setting [13]. Dosing errors are frequent, with a study of 1933 pediatric patients identifying overdosing in $8 \%$ of cases (most commonly with analgesics) and underdosing in $7 \%$ of cases (most commonly with antiepileptic medications) 
Table 1. Error types in pediatric ambulatory care

\begin{tabular}{ll}
\hline Type of error & Example of errors \\
Medication errors & Wrong drug prescribed or administered \\
Wrong dose prescribed or administered \\
Wrong route prescribed or administered \\
Wrong frequency or duration prescribed or administered \\
Omitted or duplicated dose \\
Drug-drug interaction \\
Known allergy \\
Wrong timing of vaccine \\
Diagnostic errors & Wrong route of administration \\
& Storage and dispensing errors \\
& Wrong vaccine administered \\
& Errors in the evaluation of signs and symptoms \\
Errors in follow-up of diagnostic tests \\
Errors related to missed, incorrect or delayed diagnoses \\
care transition errors & Misfiled or erroneously entered patient information \\
& Lack of consent for an adult accompanying a child \\
& Delay in office care \\
Wrong patient registered or treated
\end{tabular}

[8]. Incorrect administration by a caregiver is the most common cause of preventable adverse drug events in the ambulatory setting [14]. A study of medication errors at home in children with cancer identified 72 medication errors in 92 home visits, most commonly a parent administering a wrong dose or missing scheduled doses, with a rate of injury due to errors of 3.6\% [15]. A randomized controlled trial of over 2000 caregivers identified increased frequency of dosing errors when using dosing cups versus oral syringes, and when using milliliter measuring tools with a medication dose provided in teaspoons $[16 \bullet \bullet$. Communication failures regarding dose changes, filling prescriptions, or between caregivers have been described [17]. Medication discrepancies are common during care transitions, particularly the hospital-to-home transition [18].

\section{Strategies to support medication safety in pediatric ambulatory care}

One of the most well-studied interventions to prevent medication errors is medication reconciliation. This formal process involves compiling an accurate and comprehensive list of medications being taken by the patient (including name of medication, dose, route, and frequency), verifying with another information source (e.g., the electronic health record), and identifying and resolving any discrepancies [19]. Medication reconciliation is recommended at all transitions of care to identify potential omissions, duplications, or interactions [20]. The process is limited in that it cannot capture medication administration 
Table 2. Examples of interventions to prevent error in the ambulatory setting

\begin{tabular}{|c|c|c|}
\hline Category & Intervention & References \\
\hline \multirow[t]{4}{*}{ Medication errors } & $\begin{array}{l}\text { Standardized medication prescribing } \\
\text { and administration practices }\end{array}$ & $\begin{array}{l}\text { (AAP Committee on Drugs) } \\
\text { (CDC PROTECT Initiative) }\end{array}$ \\
\hline & Medication reconciliation & $\begin{array}{l}\text { (WH0 The High5s Project - Standard Operating } \\
\text { Protocol for Medication Reconciliation) }\end{array}$ \\
\hline & $\begin{array}{l}\text { Computerized order entry in combination } \\
\text { with clinical decision support }\end{array}$ & (Kaushal 2007) \\
\hline & Communication strategies: teach-back & (Yin 2011) (Turner 2009) \\
\hline \multirow[t]{2}{*}{ Vaccine errors } & Pharmacist integration into primary care clinic & (Haas-Gehres 2014) \\
\hline & $\begin{array}{l}\text { Prescriber education, age-specific alert } \\
\text { reminders on electronic health records }\end{array}$ & (Rogers 2016) \\
\hline Diagnostic errors & $\begin{array}{l}\text {-Improved access to electronic health records } \\
\text {-Rapid follow-up } \\
\text {-Diagnostic decision support tools }\end{array}$ & (Singh 2010) \\
\hline \multirow[t]{3}{*}{$\begin{array}{l}\text { Care coordination } \\
\text { and care transitions }\end{array}$} & $\begin{array}{l}\text { Bundled handoff interventions } \\
\text {-Standardized written and verbal } \\
\text { communication using a structured mnemonic } \\
\text {-Provider training } \\
\text {-Visual reinforcement campaign }\end{array}$ & (Starmer 2014) \\
\hline & $\begin{array}{l}\text { Care Transitions Intervention } \\
\text {-Medication self-management } \\
\text {-Patient-centered record } \\
\text {-Follow-up } \\
\text {-Identification of red flags }\end{array}$ & $\begin{array}{l}\text { (Coleman 2006) } \\
\text { (Coller 2018) }\end{array}$ \\
\hline & $\begin{array}{l}\text { Hospital-to-Home transitions intervention } \\
\text {-Transition checklist } \\
\text {-Post-discharge phone call } \\
\text {-Teachback } \\
\text {-Communication with primary care provider }\end{array}$ & (Mallory 2017) \\
\hline
\end{tabular}

errors. There has been little study of its implementation in the ambulatory pediatrics [21] and the impact on reducing adverse drug events.

To reduce opportunity for administration errors, a statement by the American Academy of Pediatrics Committee on Drugs recommended milliliter-based dosing when prescribing and administering liquid medications, and distributing dosing devices like syringes with metric markings with medications to 
encourage their use instead of household spoons [22]. The CDC PROTECT initiative has sought to standardize dosing instructions and to support safe use and storage of medications, with the goal of preventing unintentional medication overdoses $[23,24]$.

A systematic review from 2014 reported reduced prescribing errors with clinician decision support tools [12•]. Computerized order entry in combination with clinical decision support systems has been described as a potentially effective prevention strategy against adverse drug events in pediatric outpatients, particularly the use of prompts for drug allergy, drug frequency, and drug weight/dose checks [14].

One study identified that improved communication between the prescribing provider and the caregiver could have prevented the majority of preventable adverse drug events in the ambulatory setting [14]. Medication education should be tailored to caregiver health literacy [25]. Communication strategies like teach-back, or prompting the caregiver to describe in their own words, can help verify understanding although is not commonly used [26]. Providing picture-based education in addition to written instructions has been associated with reduced error in dosing with infant acetaminophen [27]. One study found decreased caregiver medication administration errors associated with use of support tools like alarms or reminders [17], suggesting that promoting these practices may be an effective error prevention strategy.

Vaccine errors may include administration (or near administration) of wrong vaccine, wrong dose of vaccine administered, or a vaccine administered at the wrong time [28•]. Older studies have suggested about a third of children receive at least one mistimed or additional vaccine $[29,30]$, although more recent studies suggest a much lower vaccine administration error rate [28, 31]. In one study at a large academic center over a period of 30 months, 10 incorrect vaccines were administered and 11 were missed or administered at the wrong time, together accounting for $9.7 \%$ of reported errors [31]. Another study that measured vaccine errors at a large academic outpatient clinic over 12 years showed a rate of $0.04 \%$ errors per vaccine administered [28].

Vaccine errors are unique in that there are national surveillance databases that track the errors related to their use and administration, although reporting is voluntary. In the USA, the Vaccine Adverse Event Reporting System (VAERS) collects vaccine adverse events, most of which are adverse drug reactions. In a recent study using the VAERS, 20,585 errors were reported over 13 years to this national database; the most common errors reported included inappropriate schedule, storage and dispensing errors (most often expired vaccine administered), and wrong vaccine administered [32]. Another study utilized the England and Wales National Reporting and Learning System to describe 1745 errors over 11 years, with the most common errors being failure of timely vaccination, wrong number of doses, wrong timing, and wrong vaccine administered [33].

\section{Strategies to support vaccine safety in pediatric ambulatory care}

A few studies have assessed the impact of interventions on vaccine error rates in ambulatory pediatrics. One study examined vaccine errors at two clinics and found that integration of a clinical pharmacist in clinic significantly decreased 
vaccine error rates [34]. Root cause analyses described in other studies identified clinic-specific approaches to prevent error, including decreasing distractions for registered nurses administering vaccines and redesigning vaccine storage rooms [31]. On a larger scale, recommendations include investing in health information technology that allows for unified immunization records and decision support tools to prevent mistiming or wrong number of vaccine doses [29, $33,35]$. A randomized controlled trial of an immunization prompting system with pediatric residents in a hospital-based clinic resulted in reduced vaccine administration errors and less missed opportunities to vaccinate [36]. A more recent study found that a combination of alerts in the electronic health record and immunization education was associated with decreased vaccine errors [37]. Additional safety checks are recommended for vaccines that are used rarely or given off-schedule [28•]. Other suggestions include lobbying organizations responsible for vaccine schedules to simplify guidelines where possible, limiting changes to guidelines unless absolutely necessary [29], and advocating for manufacturers to avoid age-specific vaccine dosing [28•].

Diagnostic errors are defined as the failure to establish an accurate and timely explanation of the patient's health problem(s) or to communicate that explanation to the patient [38] - and, typically in pediatrics, to the family. Understanding the scope of diagnostic errors in ambulatory pediatrics is challenging due to the inconsistency of classification and terminology [39]. Some studies focus on diagnostic process failures (for example, errors in evaluation of signs and symptoms or follow-up of diagnostic tests), while others investigate diagnosis label failures (including diagnoses that are missed, incorrect, or delayed) $[39,40 \bullet]$. Factors that may contribute to diagnostic errors in pediatrics include systems issues such as care fragmentation, access to resources and personnel, clinician workload, and language barriers, as well as cognitive factors related to workup and clinical assessment [41]. Data-gathering is a critical component of the diagnostic process, and in pediatrics this can be a complex task when communicating with a child about symptoms and integrating history from multiple caregivers.

Diagnostic errors and "missed opportunities for diagnosis" occur frequently in ambulatory pediatrics [40•], although it is unclear how many of these errors contribute to patient harm. In a survey of pediatricians at three tertiary care institutions, half of respondents reported that they had made 1-2 diagnostic errors in the past month, and 45\% reported 1-2 instances where a diagnostic error had harmed a patient in the past year [41]. At one large academic outpatient practice, diagnostic errors accounted for just over one fifth of total errors [31]. A pilot study of errors collected at 14 locations over 4 months showed that diagnostic errors accounted for $13 \%$ of total errors [42]. Common diagnostic errors include viral illnesses being diagnosed as bacterial illnesses and misdiagnoses (particularly related to medication side effects, appendicitis, and psychiatric disorders) [41]. One study of 25 primary care pediatric practices identified that $11 \%$ of patients with abnormal lab values and $54 \%$ of patients with elevated blood pressure had experienced a diagnostic error [40•]. One study describing diagnostic process errors identified a test delayed or not performed accounting for $12.5 \%$ of errors, delay in test results accounting for 
$2.8 \%$ of errors, and patient not given requested appointment or referral accounting for $7.4 \%$ [31].

Administrative errors account for an important subset of diagnostic errors in ambulatory medicine, as much as $21-44 \%$ [31, 42, 43]. Specific office administrative errors included misfiled or erroneously entered patient information, lack of consent for an adult accompanying a child, delay in office care, wrong patient registered or treated [31], and possible hazards in waiting room or examination room space [43].

\section{Strategies to reduce diagnostic errors in pediatric ambulatory care}

Studies examining empirical interventions to address and reduce diagnostic errors are very limited [44]; this is particularly true in ambulatory pediatrics. Among the few studies that have tested interventions to reduce diagnostic errors, most that are relevant to the ambulatory setting include test-tracking systems for ordering providers [44]. Suggested root causes of diagnostic errors include inadequate care coordination and failure to gather sufficient information through history, physical exam, or chart review [31]. Proposed interventions include a collaborative approach to test solutions and redesign practice systems [45], improving access to electronic health records, and close follow-up with patients [41]. Root cause analyses have also identified clinic-specific approaches to prevent administrative error, such as labeling specimens in the examination room to prevent specimens not being tested due to lack of labels [31].

\section{Errors related to care coordination and care transitions}

Care coordination activities and care transitions are particularly vulnerable to miscommunication and error. This may be particularly true in the ambulatory setting, where communication is typically asynchronous between geographically dispersed providers of different disciplines and clinical contexts. Ensuring the quality and safety of transitions between ambulatory care and acute care, whether the Emergency Department (ED) or hospital, has been recognized as a shared responsibility [46]. Clear communication between ED and the medical home is particularly critical when there are pending tests or the child has complex medical needs [46]. Challenges have been described with written and verbal communication between providers in the ambulatory and inpatient setting, with conflicting expectations for roles and responsibilities [47]. A recent retrospective analysis of almost 13,000 children discharged from four hospitals identified a post-discharge issue in $25 \%$ of cases, most commonly related to follow-up appointments (76\%) and medications (21\%), particularly difficulty filling prescriptions $[48 \bullet \bullet]$. In one study of patients discharged from the neonatal intensive care unit, about half of families described significant challenges with at least one barrier to care, including communicating with a clinician, scheduling appointments and filling prescriptions [49].

\section{Strategies to support care coordination and care transitions in pediatric ambulatory care}

Clinicians have been called to engage in a structured and consistent approach to communication during transitions in care [46]. Bundled handoff interventions have been associated with reductions in medical error and preventable adverse events in the inpatient setting [50]. Adaptation and implementation of structured handoffs between ambulatory care and the ED was associated with 


\section{Contextual factors}

reduced miscommunications and improved perceptions of safety [51].

Multiple studies have focused on optimizing safety surrounding transitions from the inpatient setting to ambulatory care. A recent meta-analysis assessed the impact of hospital-to-home interventions focused on provider communication, care coordination and family engagement on readmissions and postdischarge ED use [52•]. Although there was notable variability across studies, post-discharge telephone follow-up or home visits, developing a written care plan for home and contingency planning were identified as being effective strategies [52•]. One study demonstrated feasibility of a bundled transition intervention incorporating teach-back with families to confirm understanding, a transition checklist, structured provider handoff communication, and a postdischarge phone call, though no clinical outcomes were reported [53]. Postdischarge phone calls have been shown to be effective at identifying issues such as medication side effects, trouble filling medications, and changes in clinical status, though changes in rates of ED visits and readmissions were not statistically significant [54]. A health coaching intervention [55] focused on medication self-management, a patient-centered health record, timely follow-up and action plans developed surrounding hospital-to-home transitions was associated with reduced healthcare utilization [56].

Multiple patient and system factors influence the incidence of errors in ambulatory pediatrics. We highlight the impact of medical complexity, health literacy and social context, and the emergence of telemedicine on patient safety in ambulatory pediatrics.

\section{Medical complexity}

Children with special healthcare needs are at an increased risk of harm related to clinical care due to a number of factors. Children with chronic conditions often require multiple medications, which is a risk factor for medication errors [17]. In one study of children with cancer, almost half had been exposed to at least one medication error in the home [15]. Safety risks and the high prevalence of unmet care needs faced by children with medical complexity have been attributed in particular to the complex nature of care delivery [57]. Children with medical complexity have increased healthcare utilization [45], and more frequent transitions between care settings may increase opportunity for care fragmentation and error. Challenges with communication and data-gathering for children and youth with neurologic impairment may contribute to risk of diagnostic error and harm. Disparities in access to care may also impact safety, with children with developmental disabilities less likely to receive care in a medical home and the benefits of care coordination and preventative care including immunizations $[57,58]$.

\section{Health literacy and social context}

Health literacy, or the capacity to obtain and understand information to make health decisions, is central to patient safety [59]. A national survey of pediatricians identified frequent issues related to patients' understanding of health 
information and communication-related errors [26]. Health literacy plays a critical role in families' management of chronic conditions at home and in mitigating ( ${ }^{* *}$ or reducing?) risk of medical errors, particularly related to medication administration [60]. Health literacy and language barriers have also been identified as important factors in diagnostic errors [41]. Primary language and sociocultural factors may affect response rates in studies of patient safety and reporting of adverse drug events [10]. Children in foster care and children of families who have recently immigrated may be particularly vulnerable to harm related to access to care, for example, increased vaccine-related safety incidents have been described in these populations [33].

\section{Care delivery: emergence of telemedicine}

Telemedicine, or the provision of direct patient care by a provider via videoconferencing, has profoundly altered the scope and nature of ambulatory care. The American Academy of Pediatrics 2015 policy statement on telemedicine, reaffirmed in 2019, reports opportunities for this technology to improve access to timely care and quality of patient care, while cautioning the possibility of care fragmentation when care is provided outside of the medical home [61]. Telemedicine has been recognized as an effective platform for emergency response and frontline care during pandemics [62], an application that has been realized during the COVID-19 outbreak [63].

The use of telemedicine in pediatrics has increased rapidly over the past decade, most commonly for acute infections of the ear, nose, and throat [64]. Telemedicine visits have been studied in various subgroups of pediatric patients on home parenteral nutrition [65], home ventilator support [66], and for postoperative urologic follow-up [67], for example. Reported outcomes have included satisfaction, cost reduction, healthcare utilization, antibiotic prescribing and clinical outcomes such as reduced infections [65-68]. There has been limited study of the impact on patient safety outcomes. We recognize the potential of telemedicine to contribute to safety in ambulatory pediatrics, for example through ensuring regular follow-up with specialists, opportunity to observe medication administration and feeds in the home setting as well as to assess safety of the home environment. Challenges have been recognized related to training providers and the use of different telemedicine applications $[69,70]$. Studies have suggested that clinicians are able to provide appropriate diagnoses and treatment recommendations via telemedicine despite limitations to the physical examination [71]; further study of the incidence of diagnostic error and other error types should be performed.

\section{Recommendations}

Review of the literature on safety threats and sources of preventable harm in ambulatory pediatrics reveals variability in reporting and outcomes and a paucity of data specific to the ambulatory setting, making it challenging to identify clear solutions for optimizing patient safety. A number of common themes emerged as promising strategies that warrant further study. A conceptual framework for optimizing safety in ambulatory pediatrics is shown in Fig. 1. 


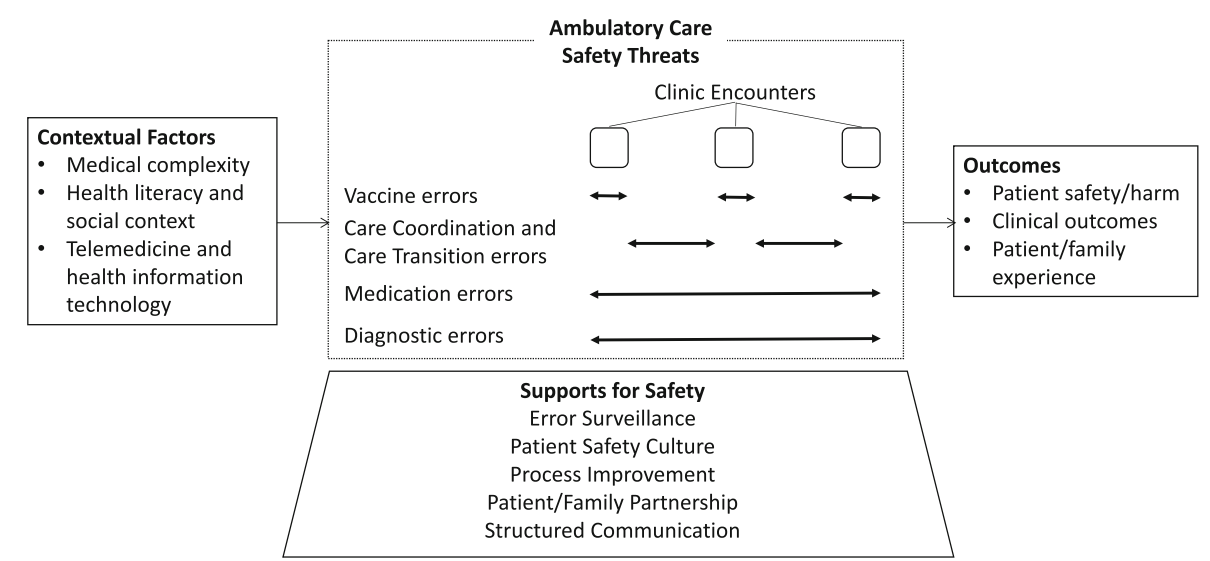

Fig. 1. Framework for patient safety in ambulatory pediatrics.

A standardized approach to surveillance in the ambulatory setting could help us to understand what types of safety incidents occur, as well as what interventions reduce error, and more importantly, reduce harm to pediatric patients. Comprehensive data collection and tracking necessitates a collaborative effort from primary and specialty providers, interprofessional care team members, administrative staff, and patients/families.

Review of the literature reveals striking inconsistencies in how safety threats are defined, categorized, and reported. Commonly accepted taxonomies include detailed classification of incidents that may be more typically seen in the inpatient setting (e.g., procedure-related incidents, hospital-acquired infections). These existing systems may not entirely reflect the nature of incidents that more commonly occur in the ambulatory setting, for example, errors in care coordination leading to miscommunications or delays in care.

Critical to the success of an effective error reporting system is educating all members of the healthcare team on what issues should be reported and providing transparency about how reported issues will be addressed.

Patient safety in the ambulatory setting relies on psychological safety within healthcare teams. Psychological safety refers to a team's shared belief that their environment supports asking for help, taking risks, and learning from mistakes [72]. A blame-free environment where individuals can report errors without fear of repercussions is central to a culture of safety in the ambulatory setting [73]. A safety culture that encourages discussion and reporting of near-miss events is necessary to identify latent safety threats [74].

There are a number of tools with validity evidence for the assessment of safety culture in ambulatory care. The medical office survey on Patient Safety Culture provided by the Agency for Healthcare Research and Quality (AHRQ) includes items on communication about error, leadership support and overall perceptions of safety and quality. The Safety Attitudes Questionnaire [75], which has been adapted to the ambulatory setting [76], explores factors including safety and teamwork climate. 
High-reliability organizations incorporate systems to anticipate and detect emerging safety threats, including an organizational climate that empowers team members to speak up for safety [77]. In order to identify and learn from near-miss events, clinicians must feel able to voice concerns or issues without risk of punishment or criticism. Establishing a voluntary, non-punitive approach in an ambulatory practice can promote increased error reporting by interprofessional team members, including patients/families [78].

\section{Partnering with patients and families for patient safety}

Partnerships with patients and families are essential to optimize safety in the ambulatory setting [79]. Partnering may involve empowering families by sharing information about potential safety threats, discussing anticipated safety issues in the home and community and collaborating to develop appropriate contingency plans. For example, optimizing medication safety through partnership includes clear discussion of medication indications and differences between dose and concentration for liquid medications; reviewing potential challenges with medication administration and performing teach-back, developing a plan for monitoring side effects, and discussing who the family should contact for medication-related issues and refills.

The critical role of families in error reporting has been described in the inpatient setting [80], yet this practice is less well-studied in ambulatory care. Discussion of the shared responsibility of providers and patients/families for preventing harm and optimizing safety should be incorporated in anticipatory guidance in routine clinic visits. Incorporating questions about safety and perceived threats to safety could be included in standard scripts for phone calls between clinic visits, particularly surrounding care transitions [79].

Beyond error surveillance, partnerships with families should involve their active participation at the ambulatory practice level. This may include development of a patient and family advisory council or integration of family partners in a quality improvement committee with a focus on safety [81]. Families as key stakeholders can provide valuable perspectives on the ambulatory care experience and help develop systems and resources to support patient safety in the home and community, through collaboration with ambulatory providers.

\section{Tools for process improvement}

Quality improvement methodology and tools for process improvement can support patient safety in the ambulatory setting [82]. Process mapping can be used to understand relationships between activities surrounding a clinic visit, for example, to understand how referrals are placed and tracked, allowing identification of any redundancy, unnecessary variation and opportunities for error. Cause and effect diagrams can help prompt team discussion of factors contributing to a safety issue in clinic. Development of SMART aims for safety initiatives and related metrics in the ambulatory setting, with regular audit and feedback of data to the interprofessional team is at the heart of supporting continuous improvement.

To know whether improvement efforts are successful at improving patient safety, clinicians need systems to track key metrics and changes over time related to the initiation of interventions. Leveraging technology to support population management through use of a patient registry may facilitate identification of atrisk subpopulations, for example, pediatric patients with polypharmacy or 
multiple missed follow-up appointments. Use of a registry or database can allow targeted approaches to ensuring safety, for example, with proactive telephone calls between visits or creating contingency plans for anticipated issues during clinic visits.

\section{Structured communication}

\section{Conclusions}

Clinicians who provide care in an ambulatory practice team can leverage evidence-based team communication strategies to optimize patient safety. The AHRQ TeamSTEPPS program [83] to develop teamwork competency has been studied in the inpatient setting, emergency departments and operating rooms, less so in ambulatory pediatrics. Potential strategies may include team huddles for developing a shared mental model of potential safety issues prior to a clinic session, highlighting needs of team members requiring additional support to ensure high-quality care in clinic, and ensuring reliable transfer of key information through closed-loop communication. Regular debriefing using a checklist to identify successes as well as opportunities for improvement can help support a proactive approach to optimizing safety. We recommend studying the impact of teamwork training and communication strategies on patient safety outcomes including miscommunications and delays in care.

Patient safety in pediatric ambulatory care remains a critical area in need of intervention and research, given the increased risk of harm due to medical care in pediatric patients generally, and the fact that the vast majority of care is delivered outside of the hospital. Interventions have been studied with promise to optimize medication safety, reduce diagnostic error and delays in care, address vaccine errors and improve communication quality across care transitions. A unification of efforts to capture and standardize how errors and harm are reported in the ambulatory setting is necessary to evaluate meaningful changes in improving patient safety. We recommend a focus on standardized error surveillance, efforts to foster safety culture, the development of strong patient/family partnerships and leveraging tools for process improvement to make headway towards a goal of high-quality, safe patient care in the ambulatory setting.

\section{References and Recommended Reading}

Papers of particular interest, published recently, have been highlighted as:

- Of importance

$\bullet \quad$ Of major importance

1. Committee on Quality of Health Care in America. In: Kohn LT, Corrigan JM, Donaldson MS, editors. To err is human: building a safer health system. Washington, DC: National Academy Press; 2000. https://doi.org/10. $17226 / 9728$

2. Neuspiel DR, Stubbs EH. Patient safety in ambulatory care. Pediatr Clin N Am. 2012;59(6):1341-54. https:// doi.org/10.1016/j.pcl.2012.08.006.

3. Hoffman JM, Keeling NJ, Forrest CB, et al. Priorities for pediatric patient safety research. Pediatrics. 
2019;143(2). https://doi.org/10.1542/peds.20180496.

4. Mohr JJ, Lannon CM, Thoma KA, et al. Learning from errors in ambulatory pediatrics. Adv Patient Saf From Res to Implement (Volume 1 Res Find. 2005. http:// www.ncbi.nlm.nih.gov/pubmed/21249808.

5. Miller MR, Robinson KA, Lubomski LH, Rinke ML, Pronovost PJ. Medication errors in paediatric care: a systematic review of epidemiology and an evaluation of evidence supporting reduction strategy recommendations. Qual Saf Heal Care. 2007;16(2):116-26. https://doi.org/10.1136/qshc.2006.019950.

6. Rood JM, Engels MJ, Ciarkowski SL, Wagenknecht LD, Dickinson CJ, Stevenson JG. Variability in compounding of oral liquids for pediatric patients: a patient safety concern. J Am Pharm Assoc. 2014;54(4):383-9.

7. Shah R, Blustein L, Kuffner E, Davis L. Communicating doses of pediatric liquid medicines to parents/caregivers: a comparison of written dosing directions on prescriptions with labels applied by dispensed pharmacy. J Pediatr. 2014;164(3):596-601. https://doi.org/ 10.1016/j.jpeds.2013.11.007.

8. McPhillips HA, Stille CJ, Smith D, et al. Potential medication dosing errors in outpatient pediatrics. J Pediatr. 2005;147(6):761-7. https://doi.org/10.1016/ j.jpeds.2005.07.043.

9. Basco WT, Garner SS, Ebeling M, Freeland KD, Hulsey TC, Simpson K. Evaluating the potential severity of look-alike, sound-alike drug substitution errors in children. Acad Pediatr. 2016;16(2):183-91. https:// doi.org/10.1016/j.acap.2015.06.014.Evaluating.

10. Zandieh SO, Goldmann DA, Keohane CA, Yoon C, Bates DW, Kaushal R. Risk factors in preventable adverse drug events in pediatric outpatients. J Pediatr. 2008;152(2):225-31.

11. Vernacchio L, Kelly JP, Kaufman DW, Mitchell AA. Medication use among children $<12$ years of age in the United States: results from the Slone survey. Pediatrics. 2009;124:446-54. https://doi.org/10.1542/peds. 2008-2869.

12. Rinke ML, Bundy DG, Velasquez CA, et al. Interventions to reduce pediatric medication errors: a systematic review. Pediatrics. 2014;134:338-60. https://doi. org/10.1542/peds.2013-3531

This systematic review assessed the effectiveness of interventions to reduce medication errors in pediatric patients. A small proportion of studies were in the ambulatory setting. Studies reported significant reductions in prescribing errors with computerized order entry with clinical decision support and preprinted order sheets. Conclusions are limited due to heterogeneity in definitions and study design.

13. Kaushal R, Goldmann DA, Keohane CA, Abramson EL, Woolf S, Yoon C, et al. Medication errors in paediatric outpatients. Qual Saf Heal Care. 2010;19(6):4-9. https://doi.org/10.1136/qshc.2008.031179.

14. Kaushal R, Goldmann DA, Keohane CA, Christino M, Honour M, Hale AS, et al. Adverse drug events in pediatric outpatients. Ambul Pediatr. 2007;7(5):3839.

15. Walsh KE, Roblin DW, Weingart SN, et al. Medication errors in the home: A multisite study of children with cancer. Pediatrics. 2013;131(5). https://doi.org/10. 1542/peds.2012-2434.

16.• Yin HS, Parker RM, Sanders LM, et al. Liquid medication errors and dosing tools: a randomized controlled experiment. Pediatrics. 2016;138(4). https://doi.org/ 10.1542/peds.2016-0357

This randomized controlled experiment assessed factors that affect medication administration errors by caregivers in the home, including labelling practices and dosing tools. More errors occurred with the use of cups versus oral syringes and with the use of teaspoon-only versus milliliter-only labels.

17. Walsh KE, Mazor KM, Stille CJ, Torres I, Wagner JL, Moretti J, et al. Medication errors in the homes of children with chronic conditions. Arch Dis Child. 2011;96(6):581-6. https://doi.org/10.1136/adc.2010. 204479.

18. Huynh C, Wong IC, Tomlin S, et al. Medication discrepancies at transitions in pediatrics: a review of the literature. Paediatr Drugs. 2013;15(3):203-15.

19. Medication Safety in Transitions of Care. 2019. http:// apps.who.int/bookorders, https://www.who.int/ patientsafety/medication-safety/technical-reports/en/.

20. The Joint Commission. National Patient Safety Goals Effective January 1, 2020. 2020.

21. Rappaport DI, Collins B, Koster A, Mercado A, Greenspan J, Lawless S, et al. Implementing medication reconciliation in outpatient pediatrics. Pediatrics.

2011;128(6):e1600-7. https://doi.org/10.1542/peds. 2011-0993.

22. American Academy of Pediatrics Committee on Drugs. Metric units and the preferred dosing of orally administered liquid medications. Pediatrics.

2015;135(4):784-7. https://doi.org/10.1542/peds. 2015-0072.

23. PROTECT Initiative: Advancing children's medication safety. Centers for Disease Control and Prevention https://www.cdc.gov/medicationsafety/protect/ protect_initiative.html. Accessed May 25, 2020.

24. Budnitz DS, Salis S. Preventing medication overdoses in young children: an opportunity for harm elimination. Pediatrics. 2011;127(6):e1597-9. https://doi.org/ 10.1542/peds.2011-0926.

25. Yin HS, Dreyer BP, Moreira HA, van Schaick L, Rodriguez L, Boettger S, et al. Liquid medication dosing errors in children: role of provider counseling strategies. Acad Pediatr. 2014;14(3):262-70. https://doi. org/10.1016/j.acap.2014.01.003.Liquid.

26. Turner T, Cull WL, Bayldon B, et al. Pediatricians and health literacy: descriptive results from a national survey. Pediatrics. 2009;124(Supplement 3):S299-305. https://doi.org/10.1542/peds.2009-1162F.

27. Yin HS, Mendelsohn AL, Fierman A, Van Schaick L, Bazan IS, Dreyer BP. Use of a pictographic diagram to decrease parent dosing errors with infant acetaminophen: a health literacy perspective. Acad Pediatr. 
2011;11(1):50-7. https://doi.org/10.1016/j.acap. 2010.12.007.

28. Reed L, Tarini BA, Andreae MC. Vaccine administration error rates at a large academic medical center and its affiliated clinics - familiarity matters. Vaccine. 2019;37(36):5390-6. https://doi.org/10.1016/j. vaccine.2019.07.027

This study measured vaccine administration errors reported to a health system's incident reporting system, yielding a rate of vaccine-related errors. Errors were found to occur more often at patient ages where few vaccines are indicated, and with vaccines that have age-specific dosing or are less commonly administered.

29. Butte AJ, Shaw JS, Bernstein H. Strict interpretation of vaccination guidelines with computerized algorithms and improper timing of administered doses. Pediatr Infect Dis J. 2001;20(6):561-5. https://doi.org/10. 1097/00006454-200106000-00003.

30. Hamlin JS, Wood D, Pereyra M, Grabowsky M. Inappropriately timed immunizations: types, causes, and their relationship to record keeping. Am J Public Health. 1996;86(12):1812-4. https://doi.org/10. 2105/AJPH.86.12.1812.

31. Neuspiel DR, Stubbs EH, Liggin L. Improving reporting of outpatient pediatric medical errors. Pediatrics. 2011;128(6). https://doi.org/10.1542/peds.20110477.

32. Hibbs BF, Moro PL, Lewis P, Miller ER, Shimabukuro TT. Vaccination errors reported to the Vaccine Adverse Event Reporting System, (VAERS) United States, 20002013. Vaccine. 2015;33(28):3171-8. https://doi.org/ 10.1016/j.vaccine.2015.05.006.

33. Rees P, Edwards A, Powell C, Evans HP, Carter B, Hibbert $P$, et al. Pediatric immunization-related safety incidents in primary care: a mixed methods analysis of a national database. Vaccine. 2015;33:3873-80. https://doi.org/10.1016/j.vaccine.2015.06.068.

34. Haas-Gehres A, Sebastian S, Lamberjack K. Impact of pharmacist integration in a pediatric primary care clinic on vaccination errors. J Am Pharm Assoc. 2014;54(2003):415-8. https://doi.org/10.1331/ JAPhA.2014.13094.Impact.

35. Evans HP, Cooper A, Williams H, Carson-Stevens A. Improving the safety of vaccine delivery. Hum Vaccines Immunother. 2016;12(5):1280-1. https://doi.org/10. 1080/21645515.2015.1137404.

36. Shaw JS, Samuels RC, Larusso EM, Bernstein HH. Impact of an encounter-based prompting system on resident vaccine administration performance and immunization knowledge. Pediatrics. 2000;105(4 II):97883.

37. Rogers J, Sebastian S, Cotton W, Pippin C, Merandi J. Reduction of immunization errors through practitioner education and addition of age-specific alerts in the electronic prescribing system. Am J Health Pharm. 2016;73(11):S74-9. https://doi.org/10.2146/ ajhp150311.
38. National Academies of Sciences Engioneering and Medicine. Improving diagnosis in health care. Washington, DC: The National Academies Press; 2015.

39. Newman-Toker DE. A unified conceptual model for diagnostic errors: underdiagnosis, overdiagnosis, and misdiagnosis. Diagnosis. 2014;1(1):43-8. https://doi. org/10.1515/dx-2013-0027.

40. Rinke ML. Diagnostic errors in primary care pediatrics: Project RedDE. Acad Pediatr. 2019.

This study assessed the frequency of diagnostic errors and missed opportunities for diagnosis in a primary care setting. It identified a high frequency of cases where recognition of elevated blood pressure, abnormal lab values and evaluation for depression was not documented.

41. Singh H, Thomas EJ, Wilson L, Kelly PA, Pietz K, Elkeeb $\mathrm{D}$, et al. Errors of diagnosis in pediatric practice: a multisite survey. Pediatrics. 2010;126(1):70-9. https:// doi.org/10.1542/peds.2009-3218.

42. Mohr JJ, Lannon CM, Thoma KA, et al. Learning from errors in ambulatory pediatrics. Agency for Healthcare Research and Quality (US); 2005. http://www.ncbi. nlm.nih.gov/pubmed/21249808. Accessed April 20, 2020.

43. Neuspiel DR, Guzman M, Harewood C. Improving error reporting in ambulatory pediatrics with a team approach. Adv Patient Saf New Dir Altern Approaches. 2008;1(Md):1-7.

44. Singh H, Graber ML, Kissam SM, Sorensen AV, Lenfestey NF, Tant EM, et al. System-related interventions to reduce diagnostic errors: a narrative review. BMJ Qual Saf. 2012;21(2):160-70. https://doi.org/10. 1136/bmjqs-2011-000150.

45. Cohen E, Berry JG, Camacho X, Anderson G, Wodchis W, Guttmann A. Patterns and costs of health care use of children with medical complexity. Pediatrics. 2012;130(6):e1463-70. https://doi.org/10.1542/peds. 2012-0175.

46. American Academy of Pediatrics Committee on Pediatric Emergency Medicine, American College of Emergency Physicians Pediatric Emergency Medicine Committee, Emergency Nurses Association Pediatric Committee. Handoffs: Transitions of care for children in the emergency department. Pediatrics. 2016;138(5). https://doi.org/10.1542/peds.2016-2680.

47. Solan LG, Sherman SN, DeBlasio D, Simmons JM. Communication challenges: a qualitative look at the relationship between pediatric hospitalists and primary care providers. Acad Pediatr. 2016;16(5):453-9. https://doi.org/10.1016/j.acap.2016.03.003.

48.• Rehm KP, Brittan MS, Stephens JR, et al. Issues identified by postdischarge contact after pediatric hospitalization: a multisite study. J Hosp Med. 2018;13(4):23642. https://doi.org/10.12788/jhm.2934

This retrospective study identified post-discharge issues and associated risk factors for children discharged from four children's hospitals. Post-discharge issues occurred frequently and most issues were related to follow-up appointments and medications. 
49. Enlow E, Herbert SL, Jovel IJ, Lorch SA, Anderson C, Chamberlain LJ. Neonatal intensive care unit to home: the transition from parent and pediatrician perspectives, a prospective cohort study. J Perinatol. 2014;34(10):761-6. https://doi.org/10.1038/jp.2014. 75 .

50. Starmer AJ, Spector ND, Srivastava R, West DC, Rosenbluth G, Allen AD, et al. Changes in medical errors after implementation of a handoff program. $\mathrm{N}$ Engl J Med. 2014;371(19):1803-12. https://doi.org/ 10.1056/NEJMsa1405556.

51. Huth K, Stack AM, Chi G, et al. Developing standardized "receiver-driven" handoffs between referring providers and the emergency department: results of a multidisciplinary needs assessment. Jt Comm J Qual Patient Saf. 2018. https://doi.org/10.1016/j.jcjq.2018. 05.003.

52. Speier RL, Vu PD, Tancredi D, et al. Hospital-to-home interventions, use, and satisfaction: a meta-analysis. 2020;142(5).

This meta-analysis assessed the effectiveness of pediatric hospital discharge interventions on healthcare utilization and satisfaction. Follow-up care, discharge planning, teach-back education and contingency planning were intervention components associated with reduced readmission rates.

53. Mallory LA, Osorio SN, Prato BS, DiPace J, Schmutter L, Soung P, et al. Project IMPACT pilot report: feasibility of implementing a hospital-to-home transition bundle. Pediatrics. 2017;139(3):e20154626. https://doi. org/10.1542/peds.2015-4626.

54. Heath J, Dancel R, Stephens JR. Postdischarge phone calls after pediatric hospitalization: an observational study. Hosp Pediatr. 2015;5(5):241-8. https://doi.org/ 10.1542/hpeds.2014-0069.

55. Coleman EA, Parry C, Chalmers S, S M. The are transitions intervention. Arch Intern Med 2006;166:18221828. doi:https://doi.org/10.1002/9781118785775. ch13.

56. Coller RJ, Klitzner TS, Lerner CF, Nelson BB, Thompson LR, Zhao Q, et al. Complex care hospital use and postdischarge coaching: a randomized controlled trial. Pediatrics. 2018;142(2):e20174278. https://doi.org/ 10.1542/peds.2017-4278.

57. Schindler CA, Pordes ES, Finkenbinder SD, Lee KJ. Safety in children with medical complexity: our canaries in the coal mine? Curr Treat Options Pediatr. 2019;5:165-82. https://doi.org/10.1007/s40746-01900159-2.

58. Cheak-Zamora NC, Thullen M. Disparities in quality and access to care for children with developmental disabilities and multiple health conditions. Matern Child Health J. 2017;21:36-44. https://doi.org/10. 1007/s10995-016-2091-0.

59. Morrison AK, Glick A, Shonna YH. Health literacy: implications for child health. Pediatr Rev. 2019;40(5):263-77. https://doi.org/10.1542/pir. 2018-0027.

60. Rothman RL, Yin HS, Mulvaney S, Co JPT, Homer C, Lannon C. Health literacy and quality: focus on chronic illness care and patient safety. Pediatrics. 2009;124:S315-26. https://doi.org/10.1542/peds. 2009-1163H.

61. Committee on Pediatric Workforce. The use of telemedicine to address access and physician workforce shortages. Pediatrics. 2015;136:202-9. https://doi.org/ 10.1542/peds.2015-1253.

62. Burke BL, Section on Telehealth Care. Telemedicine: pediatric applications. Pediatrics. 2015;136:e293-308. https://doi.org/10.1542/peds.2015-1517.

63. American Academy of Pediatrics. Guidance on Providing Pediatric Well-Care During COVID-19.

64. Ray KN, Shi Z, Poon SJ, Uscher-Pines L, Mehrotra A. Use of commercial direct-to-consumer telemedicine by children. Acad Pediatr. 2019;19(6):665-9. https://doi. org/10.1016/j.acap.2018.11.016.

65. Raphael BP, Schumann C, Garrity-Gentille S, McClelland J, Rosa C, Tascione C, et al. Virtual telemedicine visits in pediatric home parenteral nutrition patients: a quality improvement initiative. Telemed eHealth. 2019;25(1):60-5. https://doi.org/10.1089/ tmj.2017.0298.

66. Casavant DW, McManus ML, Parsons SK, Zurakowski D, Graham RJ. Trial of telemedicine for patients on home ventilator support: feasibility, confidence in clinical management and use in medical decisionmaking. J Telemed Telecare. 2014;20(8):441-9. https://doi.org/10.1177/1357633X14555620.

67. Finkelstein JB, Cahill D, Kurtz MP, Campbell J, Schumann C, Varda BK, et al. The use of telemedicine for the postoperative urological care of children: results of a pilot program. J Urol. 2019;202(1):159-63. https:// doi.org/10.1097/JU.0000000000000109.

68. Foster CB, Martinez KA, Sabella C, Weaver GP, Rothberg MB. Patient satisfaction and antibiotic prescribing for respiratory infections by telemedicine. Pediatrics. 2019;144(3). https://doi.org/10.1542/peds. 2019-0844.

69. Agboola S, Kvedar J. Telemedicine and patient safety. PSNet: Agency for Healthcare Research and Quality.

70. Olson CA, Mcswain SD, Curfman AL, Chuo J. The current pediatric telehealth landscape. Pediatrics. 2018;141(3). https://doi.org/10.1542/peds.20172334.

71. Haimi M, Brammli-Greenberg S, Baron-Epel O, Waisman Y. Assessing patient safety in a pediatric telemedicine setting: a multi-methods study. BMC Med Inform Decis Mak. 2020;20(1):1-14. https://doi.org/ 10.1186/s12911-020-1074-7.

72. Agency for Healthcare Research and Quality. Creating psychological safety in teams. EvidenceNOW Initaitive. 2018:1-2. http://www.ahrq.gov/professionals/ education/curriculum-.

73. Patient Safety Primer: Culture of Safety. PSNet: Agency for Healthcare Reserch \& Quality. https://psnet.ahrq. gov/primer/culture-safety. Published 2019. Accessed May 25, 2020.

74. Shekelle PG, Sarkar U, Shojania K, et al. Patient safety in ambulatory settings. AHRQ Publ No 16(17)- 
EHC033-EF Oct. 2016;27(27):719-725. https://www. ncbi.nlm.nih.gov/pubmedhealth/PMH0091575/pdf/ PubMedHealth_PMH0091575.pdf.

75. Sexton JB, Helmreich RL, Neilands TB, Rowan K, Vella K, Boyden J, et al. The Safety Attitudes Questionnaire: psychometric properties, benchmarking data, and emerging research. BMC Health Serv Res. 2006;6:1-10. https://doi.org/10.1186/1472-6963-6-44.

76. Modak I, Sexton JB, Lux TR, Helmreich RL, Thomas EJ. Measuring safety culture in the ambulatory setting: the safety attitudes questionnaire - ambulatory version. J Gen Intern Med. 2007;22(1):1-5. https://doi.org/10. 1007/s11606-007-0114-7.

77. Patient Safety Primer: High Reliability. PSNet: Agency for Healthcare Reserch \& Quality. https://psnet.ahrq. gov/primer/high-reliability. Published 2019. Accessed May 25, 2020.

78. Neuspiel DR, Stubbs EH, Liggin L. Improving reporting of outpatient pediatric medical errors abstract. Pediatrics. 2011;128:e1608-13. https://doi.org/10.1542/ peds.2011-0477.

79. Institute for Patient- and Family-Centered Care. Partnering with patients and families to enhance safety and quality: a mini toolkit. http://www.ipfcc.org/tools/ Patient-Safety-Toolkit-04.pdf. Published 2011. Accessed May 25, 2020.

80. Khan A, Coffey M, Litterer KP, Baird JD, Furtak SL, Garcia BM, et al. Families as partners in hospital error and adverse event surveillance. JAMA Pediatr. 2017;171(4):372-81. https://doi.org/10.1001/ jamapediatrics.2016.4812.

81. Institute for Patient- and Family-Centered Care. Advancing the practice of patient-and family-centered care in primary care and other ambulatory settings: how to get started. Institute for Patient- and Family-Centered Care. http://www.ipfcc.org/resources/getting_started. pdf. Published 2016. Accessed May 25, 2020.

82. Science of Improvement: Setting Aims. Institute for Healthcare Improvement. http://www.ihi.org/ resources/Pages/HowtoImprove/ ScienceoflmprovementSettingAims.aspx. Accessed May 25, 2020.

83. US Department of Health and Human Services, Agency for Healthcare Research and Quality. TeamSTEPPS home page. http://teamstepps.ahrq.gov/. Accessed April 20, 2017.

\section{Publisher's Note}

Springer Nature remains neutral with regard to jurisdictional claims in published maps and institutional affiliations. 